\title{
Zika virus and public health
}

\author{
Patricia Cintra Franco Schram ${ }^{1}$
}

DOI: http://dx.doi.org/10.7322/jhgd.114415

The widespread epidemic of Zika virus infection reported in 2015 in Brazil has become a serious public health problem due to association with the increased incidence of microcephaly in newborns of mothers infected with the virus.

The Zika virus is spread primarily by the Aedes aegypti mosquito, but can also be transmitted sexually from a man to his partners or from a pregnant woman to the fetus. The most common symptoms of the Zika virus disease are rash, pruritus, conjunctivitis, joint pain, headache and fever; these symptoms are generally mild and may persist for a few days to a week. Because of the problems due to intrauterine infection, especially microcephaly, pregnant women should take special precautions to avoid contamination.

The Zika virus infection manifestations are neurological problems, congenital microcephaly and other development problems in children whose mothers were infected during pregnancy. In the general population, Zika may present with Guillain-Barre syndrome, myelitis and meningo-encephalitis.

It is possible to detect the virus serology using RT-PCR during the acute phase of initial symptoms and viremia, and also in the urine, but usually by only for 3 to 14 days. Due to the non-existence of a test for commercial use, most cases are diagnosed clinically without any laboratory confirmation. In terms of laboratory diagnosis, some techniques have been described, but they are not yet available to the general population. In USA, an experimental test was released on March 30 for screening of blood donors.

Congenital intrauterine infection by the Zika virus is associated with microcephaly and fetal loss (by maternal infection during the first trimester). Be- tween March 2015 and April 2016, more than 5000 cases of microcephaly were reported among newborns in Brazil, which represents an increase of more than 20 times, if compared with the previous year. Other serious problems due to this viral infection are damage of the central nervous system, fetal growth restriction, placental insufficiency and fetal death.

We need to do prevention. Some laboratories have already expressed that they will invest in the research and production of a vaccine against the Zika virus, but the process could last for at least one or more decades before the vaccine be accessible to the population that would benefit from it. Other ways of prevention are recommended: Protection against mosquito bites must include individual and environmental measures. The population in the area of risk should wear pants and long sleeves, insect repellent, stay indoors (if possible), use mosquito nets. Those who are already contaminated and are in the acute phase should also avoid the bites of mosquitoes, to reduce the possibility of spreading the virus.

The fight against the Aedes aegypti is highly recommended, but it is hard work to be done; it needs awareness and the mobilization of the whole population to prevent the environment conducive to the development of the mosquito. However, due to the extraordinary capacity of biological adaptation of the mosquito, extinction is more difficult. Environmental measures include identify and eliminate areas where mosquitoes develops. Still waters inside and outside home are conducive to mosquito and must be eliminated.

At the moment we have at least 5000 children with microcephaly and other problems due to intrau- 
terine infection. What can we do for them? Microcephaly, regardless of the etiological cause, will manifest itself initially by a delay in the development of the infant. It will require the mobilization of parents and community (family, neighbors, friends, health professionals, and Government agencies) having everyone committed to help these children to reach their potential.

All of us, parents, hope that our children grow up healthy and become happy and financially independent adults. What can we do so all these children get there? Unfortunately, there is no simple solution. These children need early intervention, early stimulation, therapies and medical treatment. Parents, brothers, sisters and family need the empathy of the population so that they are not stigmatized and feel compelled to continue working with their children. Schools should prepare to receive these children in a few years; the curriculum probably needs to be modified, teachers need to be trained to teach children with learning styles that can challenge the status quo.

We must always remember that all children have potential. We are all individuals who have weaknesses and strengths; these children with microcephaly also have their strengths. We should en- courage these children to develop their virtues and strengths, rather than stigmatizing them as if they were completely disabled. They are not! However, they have special needs and their strengths should be discovered and worked with.

What is the solution? How much will it cost? I do not know. How to work with them? I highly recommend start early intervention and continued stimulation. And, as soon as possible, assess and recommend all appropriate therapies and medication treatment, if necessary and indicated by doctors.

We must prevent this virus infection in other pregnant women and prevent that other families have to face this difficult situation. Is better to be safe than sorry. Considering the fragile health conditions and social determinants such as poverty, added to the massive presence of the vector, and Brazil being a country susceptible to consolidation of Zika virus, the disease is characterized as a serious public health problem. The welfare demands increase, and there is a need to have actions carried out for the prevention and control of the problem. The Zika virus infection is a new reality in terms of public health, because there too many unknowns and too many potentially serious complications. 